\title{
Evaluation of Freshness of Soft Tissue Samples with Optical Coherence Tomography Assisted by Low Frequency Electric Field
}

DOI 10.17691/stm2015.7.1.09

Received November 7, 2014

A. Peña, PhD, Postdoctoral Research Fellow, The Jack Dodd Centre for Quantum Technology,

Department of Physics ${ }^{1}$;

A. Sadovoy, PhD, Research Scientist, Institute of Materials Research and Engineering (IMRE)2;

A. Doronin, PhD, Postdoctoral Research Fellow, The Jack Dodd Centre for Quantum Technology, Department of Physics';

A. Bykov, PhD, D.Tech.Sc., Postdoctoral Research Fellow, Department of Electrical Engineering, Optoelectronics and Measurement Techniques Laboratory;

I. Meglinski, PhD, Professor, The Jack Dodd Centre for Quantum Technology, Department of Physics ${ }^{1}$

${ }^{1}$ University of Otago, P.O. Box 56, Dunedin, 9054, New Zealand;

${ }^{2}$ Agency for Science, Technology and Research (A*STAR), 3 Research Link, 117602, Singapore;

3University of Oulu, P.0. Box 4500, Oulu, Fl-9000, Finland

We present an optical coherence tomography based methodology to determine freshness of soft tissue samples by evaluation of their interaction with low frequency electric field. Various biological tissues samples of different stages of freshness were exposed by low frequency electric current. The influence of the low frequency electric field on tissues was observed and quantified by the double correlation optical coherence tomography (dcOCT) approach developed in house. The quantitative evaluation of electric field - tissue interaction by dcOCT shows an increase of its relative magnitude with the tissue freshness from fresh to the non-fresh ones. The presented approach has a strong potential in food sciences for evaluation of meat quality.

Key words: optical coherence tomography; low frequency electric field; soft biological tissue samples.

Optical coherence tomography (OCT) is a non-invasive, high resolution optical imaging technique that can perform in vivo high resolution, cross-sectional microstructure imaging of biological tissues and other scattering materials [1, 2]. First successful application of OCT was demonstrated in ophthalmology, in particular, to visualize an anterior/ posterior eye segment [3] and to measure a blood flow in the retina vessels $[4,5]$. The field of OCT applications continues growing very intensively. The superior capability of imaging of the scattering media and inner flows with high spatial and time resolution allows one to conduct detailed investigations of the structure and the functioning of the blood circulatory system of humans and animals [6, 7], imaging of embryonal development [8], as well as the study of cell motility dynamics [9].

The effects of electromagnetic fields interaction with biological tissues have been studied widely in the past [10]. A number of studies have been performed to evaluate the effects of different electrical stimulation procedures to obtain tenderer and high quality meat [11]. Recently it was demonstrated that pulsed electric fields affect the moisture content of meat [12].

Recently, the double correlation OCT (dcOCT) approach was introduced and applied to observe molecular diffusion in skin tissues [13, 14], visualization vascular skin bed in vivo [15], investigate electro-kinetic response of biological tissues influenced by electric field $[16,17]$ and its changes with optical clearing [18].

In current paper, the dcOCT approach, a functional extension of conventional OCT, has been applied to study the electro-kinetic properties of tissues when a low frequency electric field is applied to tissue samples ex vivo.

Soft biological tissue samples are often used in biomedical research for validation of diagnostic methods and in vitro studies. Obviously, the freshness of tissue samples is an important issue that potentially can effect on the validity of experimental laboratory studies. Surprisingly there are no standards of freshness until now. The researchers rely on the very subjective parameters such as colour and smell of the samples. In current study we consider the application of dcOCT-based methodology to determine and/or quantify the freshness of soft tissue samples ex vivo stored at different conditions.

Materials and Methods. Recently it has been demonstrated that dcOCT is sensitive to visualize the degree of influence of the applied electric field in biological tissues [16-18]. In order to determine freshness of tissue samples, a standard swept source OCT (OCM1300SS, Thorlabs, Inc., USA) with central wavelength of $1325 \mathrm{~nm}$ and spectral bandwidth of $100 \mathrm{~nm}$ operated in polarization sensitive mode without phase retardation has been used to acquire OCT images of tissue samples ex vivo. The

Corresponding author: Igor Meglinski, e-mail: igor.meglinski@otago.ac.nz 


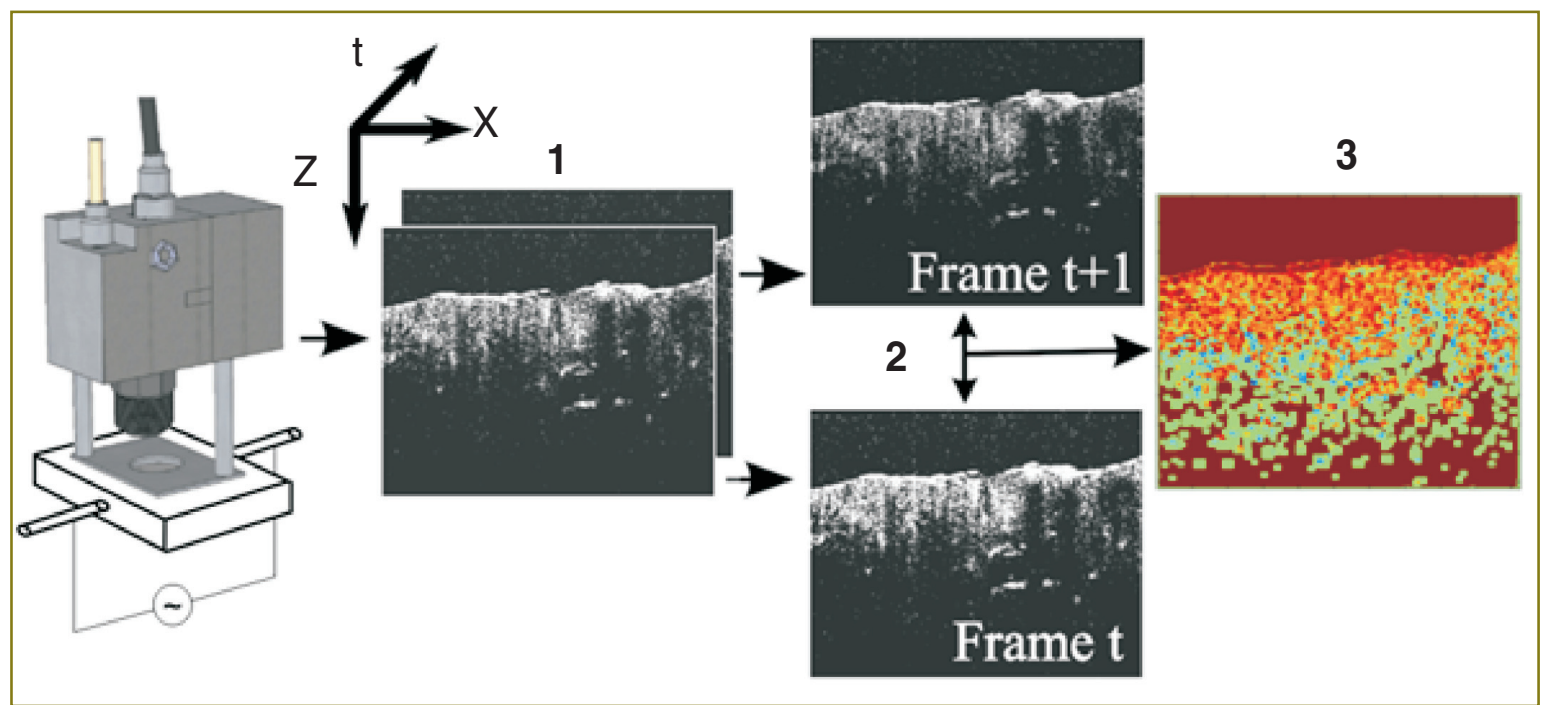

Figure 1. Schematic presentation of double correlation OCT approach. Step 1 corresponds to polarization-sensitive OCT image acquisition. Step 2 shows the Wiener filtering of the obtained images. Step 3 represents the crosscorrelation procedure between the Wiener filtering images obtained after and before the exposure of a $10 \mathrm{~V} 1 \mathrm{~Hz}$ ac current

samples are taken from fresh chicken breast pectoralis muscles and aged under different conditions: $0^{\circ} \mathrm{C}$ during 6 days, $4^{\circ} \mathrm{C}$ during 8 days, and $15^{\circ} \mathrm{C}$ during 8 days.

During the experiment, two electrodes $(\varnothing=0.3 \mathrm{~mm})$ made of stainless steel were inserted into the tissue sample towards each other at the depth of about $1 \mathrm{~mm}$ parallel to the direction of the collagen fibres. The distance between the tips of the electrodes inside the sample was of $1.5 \mathrm{~cm}$. AC electric current/voltage from function generator (GFC 2100, Iso-Tech, UK) was applied to the electrodes. The amplitude of the signal was in the range of $1-20 \mathrm{~V}$, the frequency varies within $0.1-5.0 \mathrm{~Hz}$. OCT B-scans were taken from the area between the electrodes perpendicular to the direction of current flow. To evaluate the degree of similarity of OCT images obtained after and before the exposure of the electric field the dcOCT is applied. The dcOCT approach can be summarized in 3 steps, as shown in Figure 1. Step 1 involves the acquisition of the image before and after the exposure to the electric field. Step 2 consists of the Wiener filtering, while step 3 consists of the calculation of the cross correlation image and the relative magnitude of influence of the electric field. Detailed description of dcOCT approach is given in [15].

Image processing techniques. Background noise from the experimental setup and/or sudden movements of OCT probe result imaging artefacts (such as blur, physical offsets of tissue boundaries) and, therefore, makes it quite challenging to obtain images with high spatial resolution by using the image-quality demanding correlation procedure. In the cross-correlation analysis the size of the grid should also be carefully chosen as it is a trade-off between the processing time and the final quality of the outcome [19]. If a large grid (e.g. $40 \times 40$ pixels) is used, 'blurring' and a loss of structural signal occurs [19]. For a small grid (e.g. $7 \times 7$ pixels), the background noise of OCT has a significant impact on the structural signal which results in decorellation [19]. Experimental evidence suggests that the optimal size of the grid for the biological tissues lies within the range 5-25 pixels [19].

Thus, the background noise should be suppressed. The adaptive Wiener filtering procedure removes the unwanted background noise from the images which allows better cross-correlation outcomes to be obtained for smaller grid sizes. It should be pointed out that the other techniques to eliminate the weak correlation effects could also be used (e.g. structural mask) [19].

The original implementation of the filtering procedure suggested by Wiener was applied [20]. Commonly used in image processing, Wiener filtering utilizes a sliding window (or grid) of size of $M$ by $N$ pixels centred on each pixel of the input image (Figure 2).

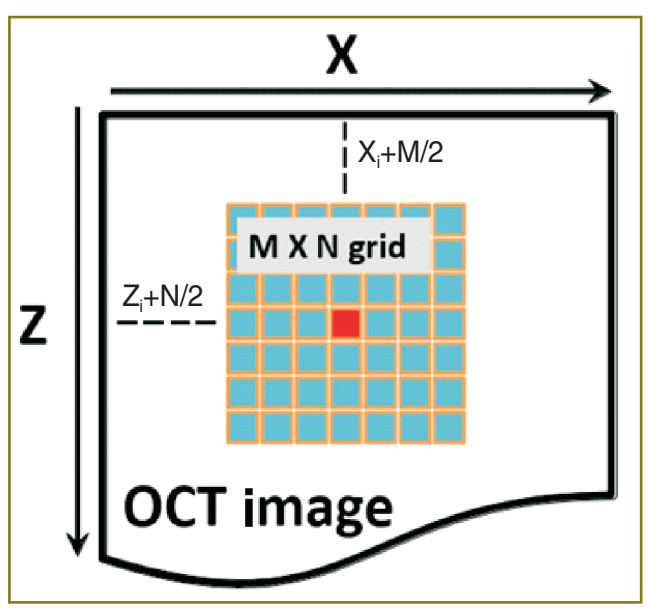

Figure 2. Schematics of Wiener filtering procedure. A grid $M \times N$ pixels is moving along the OCT image. The noise autocorrelation is estimated within the grid, subtracted from the original signal and the desired OCT image is finally obtained 
In the frequency-domain the optimal Wiener filter $W(I)$ is defined as [20, 21]:

$$
W(I)=\frac{P_{S}(I)}{P_{S}(I)+P_{N}(I)},
$$

where $P_{S}(l)$ and $P_{N}(l)$ are the power spectrums of signal and noise, respectively. $P_{S}(I)$ is obtained by taking the Fourier transform of that grid's autocorrelation according to the Wiener-Khinchin theorem [20], whereas the power spectrum of noise is estimated by calculating the local variance for each grid [21]. Thus, the desired OCT signal is obtained by subtracting an estimate of the noise from the original signal. The procedure is repeated for all grids and resulting OCT images are obtained. Figure 3 shows an example of noise removal images by the Wiener filtering.

There are a number of other approaches to create Wiener filter coefficients, including Bayesian implementation of the Wiener filter, hidden Markov models, additive noise reduction, etc. [20].

Double correlation OCT imaging works by comparing two successive OCT images that correspond to the same structural information so that one is able to map out the regions of interest. The technique performs some extra image processing steps designed to enhance the experimental data. In the correlation mapping OCT approach [19] a grid $I_{A}$ from image $A(x, z)$ is compared to the same grid $I_{B}$ from image $B(x, z)$ defined over $X-Z$ plane. The $M$ by $N$ grid is shifted pixel-by-pixel across the images, forming a correlation plane $C(x, z)$. Normalized crosscorrelation function can be written as:

$$
C(x, z)=\sum_{p=0}^{M} \sum_{q=0}^{N} \frac{I_{A}(p, q) I_{B}(p, q)}{\sqrt{I_{A}(p, q)^{2} I_{B}(p, q)^{2}}} .
$$

The output has values in the range \pm 1.0 , indicating low correlation to both high positive and high negative correlation. Direct computation of normalized crosscorrelation is very computationally expensive, time consuming and can be optimized by various techniques such as sum-tables [21]. However, more efficient approach to calculate the cross-correlation is based on the fast Fourier transform (FFT). The Fourier transform is widely used in a large class of signal processing algorithms for fast and efficient function decomposition and analysis [20]. Our approach utilizes the cross-correlation theorem, stating that the cross-correlation is equal to the inverse Fourier transform of the product between individual Fourier transforms of two functions, presuming one of them is represented by a complex conjugate [20, 21]. Thus, to find the cross-correlation between two grids the following equation is used $[20,21]$ :

$$
C(x, z)=F^{-1}\left(F\left(I_{A}\right) \times \overline{F\left(I_{B}\right)}\right) .
$$

GPU acceleration of image processing. The analysis of the images has been performed on GPUs utilizing recently developed CUDA framework in frequency-domain (Figure 4). CUDA framework gives access to the CUDA fast Fourier transform library [22]. The algorithm allows carrying out FTT computations in parallel utilizing hundreds of GPU

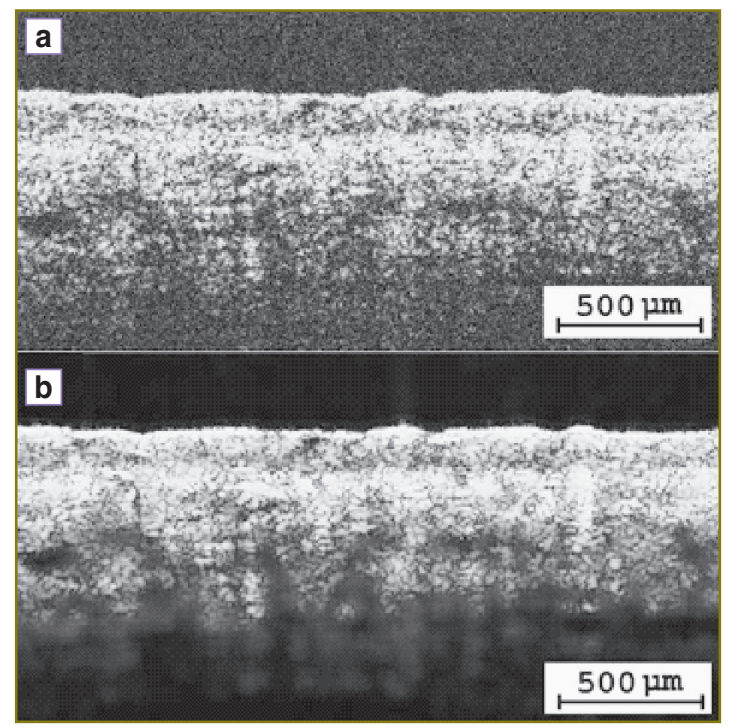

Figure 3. An example of the noise removal image by the Wiener filtering procedure from the conventional OCT image: (a) before filtering; (b) after filtering

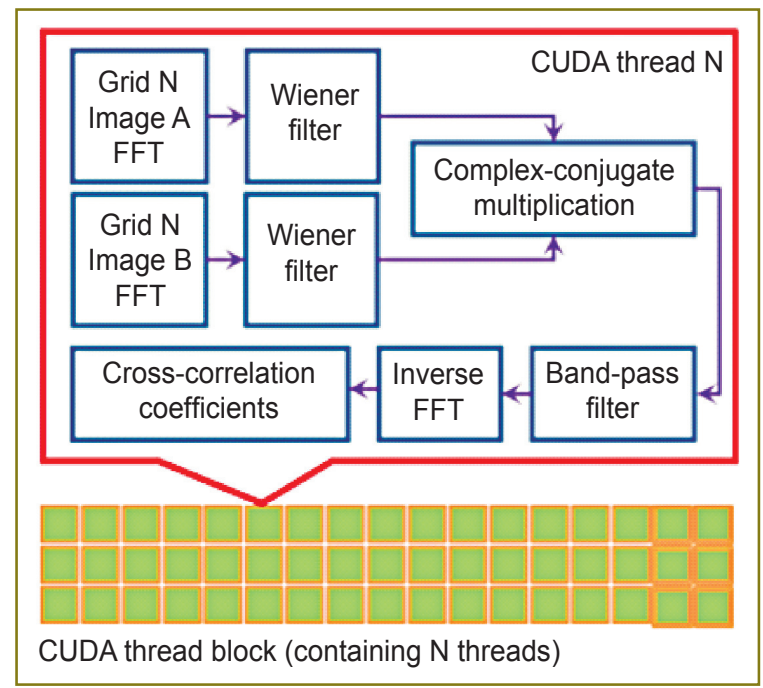

Figure 4. Schematic presentation of the double correlation OCT approach performed on GPUs

cores providing a significant speed up compared to the standard CPU approach (up to $1000 \times$ faster) [23].

It should be pointed out that if the images do not have the same dimensions, the wraparound error could occur $[20,21]$. To cancel the effects of spatial aliasing, the zero-padding procedure is typically employed: to prevent the periodic replicas from overlapping, the size of the images in the GPU memory is increased and the empty spaces are filled with zeros [22]. It is also suggested that before obtaining the Fourier transform both images are zeromapped [20-22]. The procedure ensures that the sum of all pixels is zero and the maximum of the correlation function corresponds to the right position in the image instead of the brightest pixel [20, 21].

Moreover, the software development kit provided by the device manufacturer (Thorlabs, Inc., USA), allows 
bypassing the step of manual acquisition of the OCT images and provides the opportunity for direct export of the OCT hardware FFT-encoded image data to the processing software. Incorporating this ability in the dcOCT method significantly improves the quality of imaging outcomes and speeds up the image processing (up to 30-40 times, i.e. makes it possible to proceed in real time).

Results and Discussion. The soft biological tissues at different stages of freshness and corresponding conventional OCT images are presented in Figure 5. As one can see no changes in colour and in structural integrity correlating with the sample freshness can be observed and/ or distinguished.

To distinguish the samples of biological tissues in order of their freshness we apply dcOCT approach presented above. Figure 6 (a) shows an example of typical OCT image of chicken sample aged at $15^{\circ} \mathrm{C}$ during 8 days, while Figure 6 (b) corresponds to cross correlation image calculated using the dcOCT approach. At both figures the arc-shaped pattern corresponds to the reflection from the surface of electrode.
The relative magnitude of influence of the low-frequency external electric field (See Figure 6 (b)) is counted based on the dcOCT image as [17]:

$$
\Psi=1-\frac{1}{M \times N} \sum_{x=0}^{M-1} \sum_{z=0}^{N-1} C(x, z)
$$

Further, we investigated the changes of this relative magnitude vs the tissue samples freshness. Figure 7 shows the results of the calculation of the relative magnitude of influence of the electric field in chicken meat samples. As one can see fresh tissue has the lower relative magnitude and it follows a trend in which the non-fresh tissue stored at $15^{\circ} \mathrm{C}$ during 8 days has the highest relative magnitude. This suggests that the evaluation of the relative magnitude of influence of the electric field can be used to assess relative freshness of soft biological tissues (here, we present only the results for chicken breast tissues for the purpose of brevity, but similar results were also observed for other soft biological tissues).

We suggest that the observed behaviour is attributed

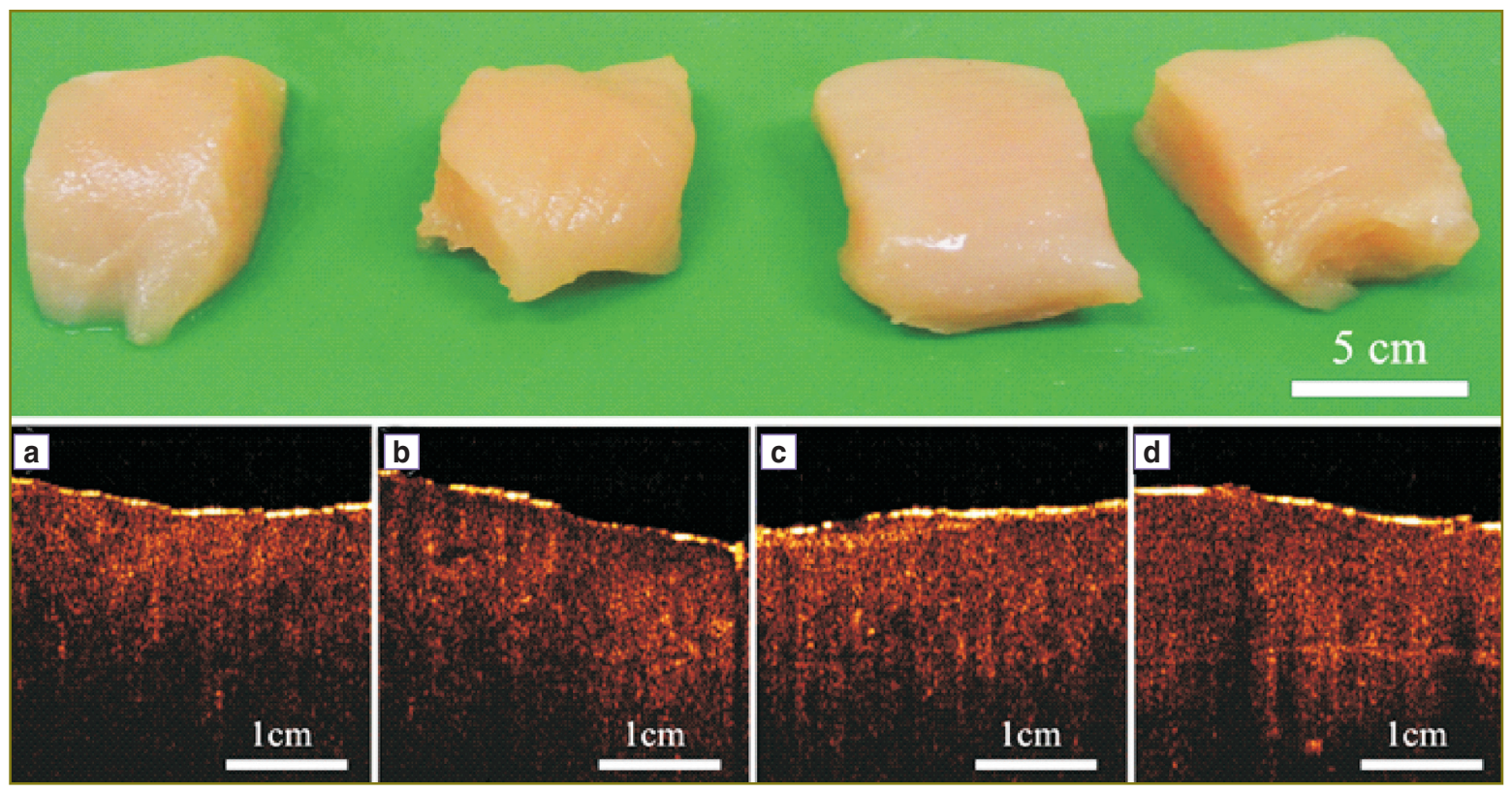

Figure 5. The samples of chicken breast at different stages of freshness (upper row) and corresponding OCT images (lower row): (a) fresh sample; (b)-(d) respectively correspond to samples stored at $0^{\circ} \mathrm{C}$ during 6 days, $4^{\circ} \mathrm{C}$ during 8 days, and $15^{\circ} \mathrm{C}$ during 8 days

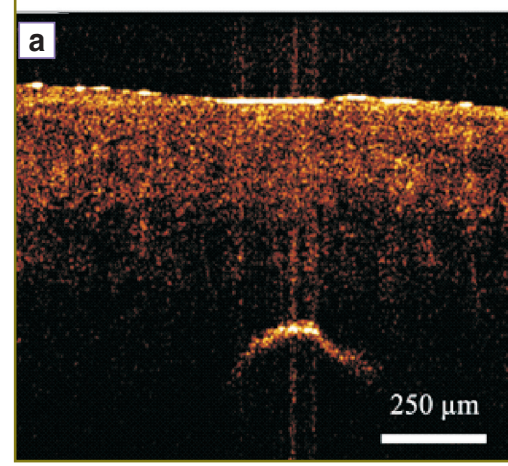

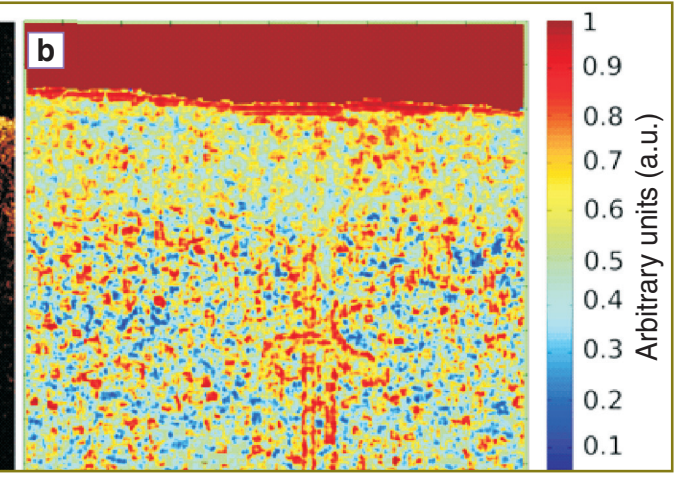

Figure 6. Typical images of soft biological tissue samples exposed with an electric field obtained by conventional OCT (a) and by dcOCT approach (b) 


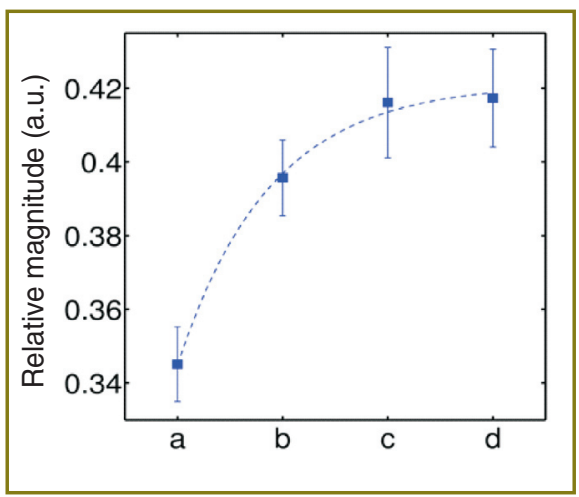

Figure 7. Relative magnitude of influence of the external electric field on the soft biological tissue samples of various freshness: $a-$ corresponds to the fresh sample; $b-d-$ respectively correspond to the samples stored at $0^{\circ} \mathrm{C}$ during 6 days, $4^{\circ} \mathrm{C}$ during 8 days, and $15^{\circ} \mathrm{C}$ during 8 days. Dashed lines correspond to the results of the inverse exponential fitting

to the tissue tendering process. The tenderness increases due to natural enzymatic changes that take place in tissue samples. Less tender tissue results in high correlation which itself reduces the amount of affectation of the electric field.

Conclusions. The obtained results show that freshness of soft biological tissues affects the propagation of ac current in the samples that can be observed with dcOCT. It has been shown that the relative magnitude of influence of the low-frequency external electric field on the biological tissue $(\Psi)$ increases from fresh to non-fresh samples as an inverse exponential function. This potentially can be used to build a phenomenological model to assess quantitatively the freshness of soft biological tissues. The possibility of real-time data processing essential for the real-life application was also demonstrated. We foresee the further development of the approach in applications in modern biomedicine as well as in the food industry.

Study Funding. This study was partially supported by the Agency for Science, Technology and Research ( $A^{*}$ STAR), Singapore, Nutrition and Food Science Grant FN-063 1121770041.

Conflict of Interests. The author declares no conflict of interest.

\section{References}

1. Optical coherence tomography: technology and applications. Drexler W., Fujimoto J. (editors). Berlin, Heidelbeg: Springer; 2008; 1346 p., http://dx.doi.org/10.1007/978-3-54077550-8.

2. Handbook of optical coherence tomography. Bouma B., Tearney G. (editors). New York: Marcel Dekker; 2002; 740 p.

3. Yasuno Y., Hong Y., Makita S., Yamanari M., Akiba M., Miura M., Yatagai T. In vivo high-contrast imaging of deep posterior eye by $1-\mu \mathrm{m}$ swept source optical coherence tomography and scattering optical coherence angiography. Opt Express 2007; 15: 6121-6139, http://dx.doi.org/10.1364/ OE.15.006121.
4. Wang Y., Bower B.A., Izatt J.A., Tan O., Huang D. In vivo total retinal blood flow measurement by Fourier domain Doppler optical coherence tomography. J Biomed Opt 2007; 12(4): 041215, http://dx.doi.org/10.1117/1.2772871.

5. White B.R., Pierce M.C., Nassif N., Cense B., Park B.H., Tearney G.J., Bouma B.E., Chen T.C., de Boer J.F. In vivo dynamic human retinal blood flow imaging using ultra-highspeed spectral domain optical coherence tomography. Opt Express 2003; 11(25): 3490-3497, http://dx.doi.org/10.1364/ OE.11.003490.

6. Bezerra H.G., Costa M.A., Guagliumi G., Rollins A.M., Simon D.I. Intracoronary optical coherence tomography: a comprehensive review: clinical and research applications JACC Cardiovasc Interv 2009; 2(11): 1035-1046, http://dx.doi. org/10.1016/j.jcin.2009.06.019.

7. Srinivasan V.J., Mandeville E.T., Can A., Blasi F., Climov M., Daneshmand A., Lee J.H., Yu E., Radhakrishnan H., Lo E.H., Sakadžić S., Eikermann-Haerter K., Ayata C. Multiparametric, longitudinal optical coherence tomography imaging reveals acute injury and chronic recovery in experimental ischemic stroke. PLoS ONE 2013; 8(8): e71478, http://dx.doi.org/10.1371/journal.pone.0071478.

8. Bhat S., Larina I.V., Larin K.V., Dickinson M.E., Liebling M. 4D reconstruction of the beating embryonic heart from two orthogonal sets of parallel optical coherence tomography slice-sequences. IEEE Trans Med Imaging 2013; 32(3): 578-588, http://dx.doi.org/10.1109/ TMI.2012.2231692.

9. Bykov A.V., Priezzhev A.V., Lauri J., Myllylä R. Doppler OCT imaging of cytoplasm shuttle flow in Physarum polycephalum. J Biophotonics 2009; 2(8-9): 540-547, http:// dx.doi.org/10.1002/jbio.200910057.

10. Polk C., Postow E. Handbook of biological effects of electromagnetic fields. CRC Press; 1986; 503 p.

11. Cetin O., Bingol E.B., Colak H., Hampikyan H. Effects of electrical stimulation on meat quality of lamb and goat meat. Scientific World Journal 2012; 2012: 574202, http://dx.doi. org/10.1100/2012/574202.

12. Faridnia F., Bekhit A.El-Din.A., Niven B., Oey I. Impact of pulsed electric fields and post-mortem vacuum ageing on beef longissimus thoracis muscles. International Journal of Food Science \& Technology 2014; 49(11): 2339-2347, http:// dx.doi.org/10.1111/ijfs.12532.

13. Kamali T., Doronin A., Rattanapak T., Hook S., Meglinski I. Assessment of transcutaneous vaccine delivery by optical coherence tomography. Laser Phys Lett 2012; 6(8): 607-610, http://dx.doi.org/10.7452/lapl.201210046.

14. Rattanapak T., Birchall J., Young K., Ishii M., Meglinski I., Rades T., Hook S. Transcutaneous immunization using microneedles and cubosomes: mechanistic investigation using optical coherence tomography and two-photon microscopy. J Controlled Release 2013; 172(3): 894-903, http://dx.doi. org/10.1016/j.jconrel.2013.08.018.

15. Doronin A., Meglinski I. Imaging of subcutaneous microcirculation vascular network by double correlation optical coherence tomography. Las \& Photon Rev 2013; 7(5): 797-800, http://dx.doi.org/10.1002/lpor. 201200108.

16. Wawrzyn K., Demidov V., Vuong B., Harduar M.K., Sun C., Yang V.X.D., Doganay O., Toronov V., Xu Y. Imaging the electro-kinetic response of biological tissues with optical coherence tomography. Opt Lett 2013; 38(14): 2572-2574, http://dx.doi.org/10.1364/OL.38.002572. 


\section{NOVEL STUDIES}

17. Peña A.F., Devine J., Doronin A., Meglinski I. Imaging of the interaction of low frequency electric fields with biological tissues by optical coherence tomography. Opt Lett 2013; 38(14): 2629-2631, http://dx.doi.org/10.1364/ OL.38.002629.

18. Peña A.F., Doronin A., Tuchin V.V., Meglinski I. Monitoring of interaction of low-frequency electric field with biological tissues upon optical clearing with optical coherence tomography. J Biomed Opt 2014; 19(8): 086002, http://dx.doi. org/10.1117/1.JBO.19.8.086002.

19. Jonathan E., Enfield J., Leahy M.J. Correlation mapping method for generating microcirculation morphology from optical coherence tomography (OCT) intensity images.
J Biophotonics 2011; 4(9): 583-587, http://dx.doi.org/10.1002/ jbio.201000103.

20. Gonzalez R., Woods R. Digital image processing. 3rd ed. Upper Saddle River (NJ): Prentice Hall; 2008; 976 p.

21. Lim J. Two-dimensional signal and image processing. Upper Saddle River (NJ): Prentice Hall; 1989; 694 p.

22. Kirk D. Hwu W. Programming massively parallel processors: a hands-on approach. Burlington (MA): M.K. Publishers; 2010; $280 \mathrm{p}$.

23. Doronin A., Meglinski I. Online object oriented Monte Carlo computational tool for the needs of biomedical optics. Biomed Opt Express 2011; 2(9): 2461-2469, http://dx.doi. org/10.1364/BOE.2.002461. 